\title{
Exploring potential cosmic ray accelerators with neutrinos: what do we learn by injecting nuclei in Gamma-Ray Bursts?
}

\author{
Denise Boncioli*, Daniel Biehl, Anatoli Fedynitch, Walter Winter \\ DESY, Platanenallee 6, 15738 Zeuthen, Germany \\ E-mail: denise.boncioliedesy.de
}

\begin{abstract}
Neutrino stacking analysis constrains the paradigm that Gamma-Ray Bursts (GRBs) are the sources of the Ultra-High Energy Cosmic Rays (UHECRs). However, most of the literature has focused on a pure proton composition of UHECRs, which has been disfavored by recent composition measurements of the Pierre Auger Observatory. The injection of nuclei in the sources is here considered by simulating the nuclear cascade in the photon field of a GRB within the (one-zone) internal shock model. We demonstrate that the prompt neutrino flux hardly depends on the injected composition and is potentially able to strongly constrain UHECR models. The cosmogenic neutrino flux expected from cosmic rays escaped from the sources is also computed: nucleons from the disintegration chain in the GRB lead to a significant contribution to this flux, altering the usual expectation related to the heavy composition at injection. A parameter space study within this combined source-propagation model is performed through a fit of the spectrum and composition data of the Pierre Auger Observatory, showing the power of this tool on constraining the features of GRBs or other classes of candidate sources of neutrinos and UHECRs.
\end{abstract}

35th International Cosmic Ray Conference - ICRC2017

10-20 July, 2017

Bexco, Busan, Korea

* Speaker. 


\section{Introduction}

The ultra-high energy cosmic rays have uncertain origin and consitute one of the most important unsolved issues in astroparticle physics. Their extragalactic origin is supported by the fact that the Galactic magnetic field would be unable to confine them, since their energy is larger than $10^{18} \mathrm{eV}$. One of the candidate sources are the Gamma-Ray Bursts [1]. They are observed as $\gamma$ ray sources lasting from a fraction of a second to hundreds of seconds. A well-accepted model to describe GRBs is the fireball model, in which the GRB itself is expected to be produced by the dissipation of the kinetic energy within an ultra-relativistic flow. During the prompt phase of the GRB, $\gamma$-ray emission is expected to be crated by internal shocks. If some contamination of the fireball by hadrons is present, a significant flux of high-energy neutrinos is expected because of the interactions of these particles with the target photons of the prompt phase [2].

In the present work, based on [3], we investigate the internal shock model, in which the considered parameters are assumed to be the same for all collisions. Other models than the internal shock model have been considered in literature, but they usually take into account only pure proton composition. Since there is evidence of a mass composition of cosmic rays heavier than protons [4] we consider the injection of nuclei in GRBs and compute the interactions in collisions of alike GRB shells. In order to test if GRBs can be the sources of cosmic rays and neutrinos, a combined sourcepropagation model is needed: the accelerated nuclei are injected in the radiation zone, where the secondaries are produced, escape from that and propagate through the extragalactic space to Earth. We perform parameter-space studies in order to directly connect the characteristics of the source to the description of the UHECR data and neutrino limits.

\section{Methods and inputs from nuclear physics and astrophysics}

The results here presented, relative to the interactions in the source, are based on the NeuCosmA code, following the implementation for protons in GRBs $[5,6,7,8]$. The extensions for nuclei have been reported in [3,9]. We choose all potentially relevant, known isotopes with $A \leq 56$. These isotopes are shown as boxes in the left panels of Fig. 1 as a function of $N$ and $Z$.

For $8 \mathrm{MeV} \lesssim \varepsilon_{r} \lesssim 150 \mathrm{MeV}$, (where $\varepsilon_{r}$ is the energy of the photon in the nucleus rest frame), the "giant dipole resonance" (GDR) and other processes lead to an electromagnetic excitation of the primary nucleus with the emission of one or more nucleons (or light nuclei). A frequently used model for the computation of photo-disintegration is the Puget-Stecker-Bredekamp (PSB) model $[10,11]$, that relies on choosing one isotope for each mass number $\mathrm{A}$, and a unique disintegration chain populated through subsequent emission of nucleons. A more sophisticated approach (used in the present work for the interactions in the source), based on the TALYS nuclear reaction program [12], allows the photo-disintegration in nucleons and small fragments and considers 481 isotopes below ${ }^{56} \mathrm{Fe}$ and about 41000 inclusive channels. The implications of different choices of cross section models on quantities relevant for cosmic-ray and neutrino physics are discussed in $[9,13,14]$. For $\varepsilon_{r} \gtrsim 150 \mathrm{MeV}$, higher energy processes, such as baryonic resonances, dominate the disintegration, accompanied by meson production. An automated isotope selection scheme is used imposing thresholds on the lifetimes of the unstable nuclei and on the multiplicity of the photo-disintegration channels in the nuclear cascade. 
The spectrum for long-duration GRBs can be typically described by a broken power law. In order to compute the photon density in the shock rest frame, we define an "isotropic volume" of the interaction region $V_{\text {iso }}^{\prime}=4 \pi R^{2} \cdot \Delta d^{\prime}$ with shell width $\Delta d^{\prime}$ and the radius (distance from emitter) of the emission region $R$. Because of the intermittent nature of GRBs, the total fluence is typically assumed to come from $\Delta T / t_{v}$ such interaction regions, where $\Delta T$ is the duration of the prompt emission and $t_{v}$ is the variability timescale, a global property of the burst's light-curve. The energy density is then $\propto L_{\gamma} /\left(4 \pi c \Gamma^{2} R^{2}\right)$, where $L_{\gamma}$ is the isotropic equivalent luminosity in gamma-rays and $\Gamma$ is the Lorentz boost factor. In the internal shock model scenario the shells of plasma are assumed to collide at a radius $R \simeq 2 \Gamma^{2} c t_{v} /(1+z)$ and the variability timescale is assumed to be indicative for the shell width $\Delta d^{\prime} \simeq \Gamma c t_{v} /(1+z)$. All collisions are assumed to collide at same radius in the one-zone model. Our results will be shown as a function of $L_{\gamma}$ and $R$ for one collision only.

Nuclei of species $i$ are assumed to be injected with a cut-off power law. The maximal energy $E_{i, \max }^{\prime}$ is determined automatically by balancing the acceleration rate $t_{\text {acc }}^{\prime-1}=\eta c / R_{L}^{\prime}$ (where $R_{L}^{\prime}$ is the Larmor radius) with the sum of synchrotron loss, adiabatic cooling, photo-disintegration, and photo-meson production rates, where we choose $\eta=1$ for the acceleration efficiency. In order to define a "nuclear loading" $\xi_{i}$ of species $i$, we take into account the optically thick case to $A \gamma$ interactions and normalize the injection luminosity to the $\gamma$-ray luminosity.

In order to compute the "direct" escape from the source, we follow Ref. [7]. Protons and nuclei can escape with a rate $\propto R_{L}^{\prime} \propto E^{\prime}$, which means that they can escape freely when/if the Larmor radius reaches the shell width. This situation can be typically found in sources where the radiation densities and the maximal primary energy are low. If, on the other hand, the radiation densities are high such that the maximal energy is limited by the photohadronic interactions, the escape at the highest energy will be suppressed.

Once the accelerated particles are able to escape from the source, they propagate through the extragalactic space, encountering photon fields, such as the Cosmic Microwave Background (CMB) and the Extragalactic Background Light (EBL, from infrared to ultraviolet), with which they interact. The dominant processes for nuclei are photo-disintegration on CMB at the highest energies and on EBL at intermediate energies, while the photo-meson production is shifted towards $A$ times the threshold for protons. At the lowest energies, nuclei lose energy adiabatically because of the expansion of the Universe. The propagation of cosmic rays ejected from the GRBs is computed using the simulation code SimProp [15] with the PSB model for photo-disintegration processes. The nuclei ejected from the source are then grouped and the corresponding fluxes are summed, assigning the sum to a mass chosen as representative for each group. The ejected particles are propagated through the extragalactic space: for this study we use the Gilmore EBL [16]. Neutrinos produced during the propagation are also computed.

\section{Nuclear-cascade source classes and neutrinos}

We describe the nuclear cascade and the characteristics of the ejected cosmic rays and neutrinos by discussing three representative cases. We inject a pure composition of ${ }^{56} \mathrm{Fe}$ only.

We define the Empty Cascade as the case which is optically thin to $A \gamma$ interactions of the injection isotope (and, consequently, all lighter isotopes including nucleons). The nuclear cascade (integrated energy of isotopes relative to total injection energy) is shown in the upper left panel of 

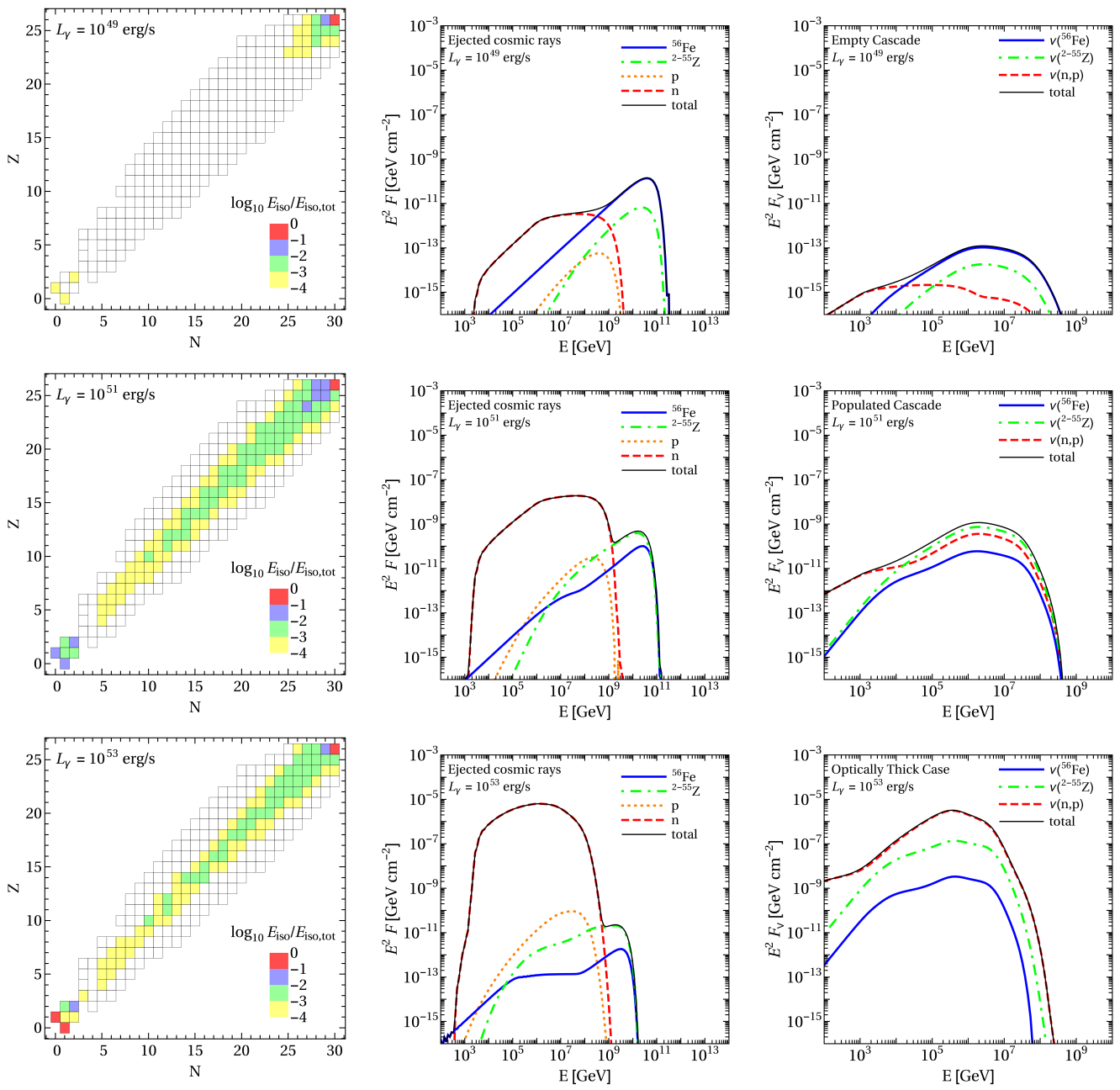

Figure 1: Figure taken from [3] - Nuclear cascade (left), ejected cosmic ray fluence per shell (center) and neutrino fluence per shell (right) as a function of the energy in the observer's frame, for the injection of pure ${ }^{56} \mathrm{Fe}$. First row: Example for the "Empty Cascade" source class (isotropic luminosity $L_{\gamma}=10^{49} \mathrm{erg} \mathrm{s}^{-1}$ ). Second row: Example for the "Populated Cascade" source class $\left(L_{\gamma}=10^{51} \mathrm{erg} \mathrm{s}^{-1}\right)$. Third row: Example for the "Optically Thick Case" (to nucleons and nuclei) source class $\left(L_{\gamma}=10^{53} \mathrm{erg} \mathrm{s}^{-1}\right.$ ). The different curves in the central panels correspond to the different isotopes, according to legend. The different curves in the right panels show the contribution of the photo-meson production off the primary $\left({ }^{56} \mathrm{Fe}\right)$ and secondary isotopes produced in the photo-disintegration, where the proton/neutron contribution is separated. The other GRB parameters are: $R \simeq 10^{8.3} \mathrm{~km}, \Gamma=300, \xi_{\mathrm{Fe}}=10, \varepsilon_{\gamma, \mathrm{br}}^{\prime}=1 \mathrm{keV}$ and $z=2$.

Fig. 1. Apart from ${ }^{56} \mathrm{Fe}$, a few closeby isotopes are populated, while most of the nuclear cascade is, relative to the injection luminosity, empty. Nucleons and light nuclei (especially ${ }^{4} \mathrm{He}$ ) are produced as disintegration products, but their occupation is relatively small compared to the injection isotope. The ejected cosmic rays are shown in the upper central panel of Fig. 1: charged cosmic rays escape via direct escape, leading to harder spectra with respect to the one for neutrons. The neutrino flux is dominated by photomeson production off the injection isotope, as shown in the upper right panel of Fig. 1.

The Populated Cascade is defined as the case which is optically thick to $A \gamma$ interactions of the 


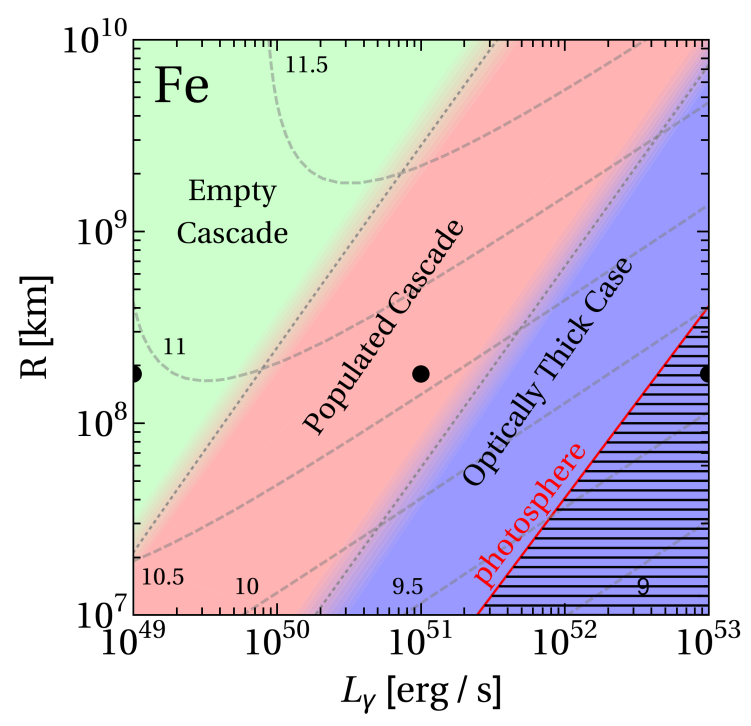

Figure 2: Figure taken from [3] - Nuclear cascade class as a function of luminosity $L_{\gamma}$ and collision radius $R$ in the internal shock scenario, for pure ${ }^{56} \mathrm{Fe}$ at injection. The other parameters are the same as in Fig. 1. Black dots indicate the position of the examples shown in Fig. 1. The gray dashed contours display $\log _{10}\left(E_{i, \max }[\mathrm{GeV}]\right)$ in the observer's frame. Note that in this figure we scale $t_{v} \propto R$.

injection isotope that will disintegrate and populate the cascade, as shown in Fig. 1, central left panel. At the same time, the source environment is optically thin to $p \gamma$ interactions, so that the nucleon fluxes will be hardly affected by these interactions. The maximal primary energy is given by $A \gamma$ interactions in this case. The ejection spectrum including neutrons and nuclei (Fig. 1, central panel) is softer than the previous case because of the neutron escape component. The neutrino flux is dominated by photomeson production off the secondary isotopes (Fig. 1, central right panel).

We define the Optically Thick Case as the one which is optically thick to $A \gamma$ interactions of nucleons and nuclei. The cascade (Fig. 1, lower left panel) appears to be less populated off the main diagonal and most energy is dumped into nucleons produced in the cascade - which are populated similarly to the primaries. The maximal primary energy is determined by photo-disintegration and the neutrino production is dominated by the protons and neutrons produced in the disintegration chain, that dominate the ejected cosmic rays (Fig. 1, lower central panel).

Fig. 2 shows the three regimes as a function of $L$ and $R$ for the injection of pure iron, where also the sub-photospheric region is reported, i.e., the region from which photons cannot escape because of Thomson scattering. The Optically Thick Case and even the photosphere are reached in the lower right corners of the panel, where the photon density is high, whereas the cascade is hardly populated in the upper left corner. The maximal primary energy (gray-dashed contours) is typically given by $A \gamma$ interactions (lower right) or adiabatic cooling (upper left). Note that the adiabatic cooling limited case corresponds to a rigidity-dependent maximal energy, and that it roughly coincides with the Empty Cascade case.

In [3] we also investigate the impact of the injection isotope (for pure composition at injection) in the neutrino flux. The flux at the peak hardly depends on the injection composition and it is mostly related to the shell parameters. As a consequence, the neutrino stacking bounds on GRBs for the case of protons apply as well in the case of nuclei.

\section{Description of cosmic-ray data}

We consider here a population of identical GRBs, following the star formation rate for the 


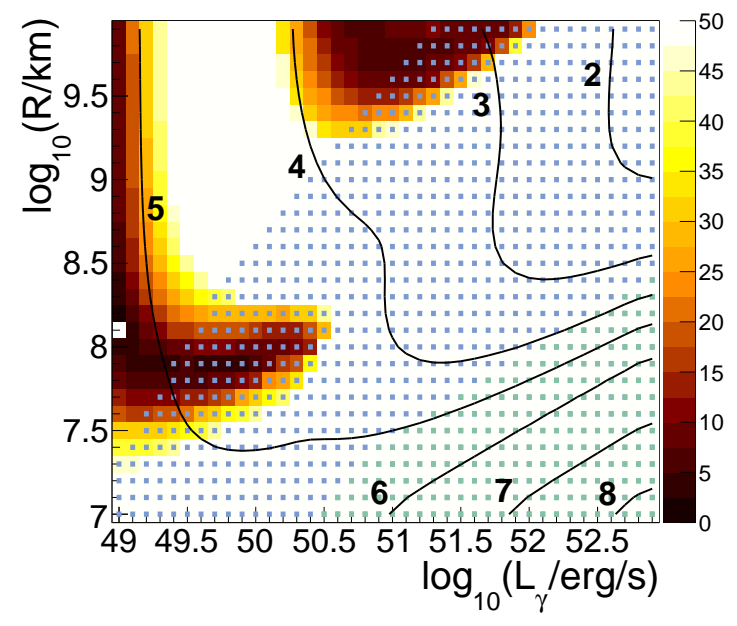

Figure 3: Figure taken from [3] - Parameter space scan for Mixed Composition Ankle Model in the internal shock scenario. Here $\sqrt{\chi^{2}-\chi_{\min }^{2}}$ is shown as a function of $R$ and $L_{\gamma}$ for the fit to cosmic-ray data of the Pierre Auger Observatory $[19,4]$ above $10^{19} \mathrm{eV}$, including a penalty for the overshooting of the flux at lower energies. The blue squares mark the current ( $90 \%$ C.L.) IceCube-excluded region from the GRB stacking analysis from Northern and Southern sky muon tracks [20,21], while the green ones mark the current (90\% C.L.) IceCube-excluded region from the cosmogenic neutrino analysis [22], applied to $v_{\mu}+\bar{v}_{\mu}$. The contours show the nuclear loading $\left(\log _{10} \xi\right)$.

source evolution, using the single-collision zone already discussed. A fit of UHECRs as measured by the Pierre Auger Observatory is performed, combining the modeling of interactions in the source with the propagation of cosmic rays in the extragalactic space. For the propagation the SimProp code is used with the details given in Sec. 2 . A pure ${ }^{28} \mathrm{Si}$ at the injection is assumed, as inspired by $[17,18]$, with a power-law index of $k=1.8$ and an exponential cutoff. The fit is performed above $10^{18} \mathrm{eV}$ (Mixed Composition Dip Model) and above $10^{19} \mathrm{eV}$ (Mixed Composition Ankle Model) by using the combined spectrum [19] and the shower depth $\left(X_{\max }\right)$ distributions [4], with a similar procedure as used in [17]. A scan over $\left(R, L_{\gamma}\right)$ is performed and for each pair the normalization to the experimental flux is found. For each point of the parameter space the number of expected prompt and cosmogenic neutrino events is calculated following [6] and the details of the calculations of the exclusion region are given in [3].

Here we show the results for the "Mixed Composition Ankle Model" (Fig. 3), which describes the UHECR data only above the ankle and a different population of sources is expected to dominate at lower energies. The goodness of fit is here enhanced with respect to the "Mixed Composition Dip Model" (describing the UHECR data above $10^{18} \mathrm{eV}$, reported in [3]). The best fit is found at low source luminosity and intermediate collision radius $\left(R=10^{8.1} \mathrm{~km}, L_{\gamma}=10^{49} \mathrm{erg} / \mathrm{s}\right)$, corresponding to the Empty Cascade. These parameters are not excluded by the existent neutrino limits, as one can also see in the upper right panel of Fig. 4. The CR observables corresponding to the best fit are also shown in Fig. 4. A mixed injection composition and the inclusion of systematic errors would improve the agreement with the data, in particular with the composition observables. However, the results that we show here can be considered as indicative for the neutrino bounds.

\section{Conclusions}

One possible class of sources for UHECRs are GRBs and have been investigated with stacking analyses, by looking at the neutrino emission from the prompt phase of GRBs, using proton primaries. Here we have studied the UHECR paradigm for GRBs in presence of nuclei, since there is evidence of CR composition heavier than hydrogen from the measurements of the Pierre Auger 

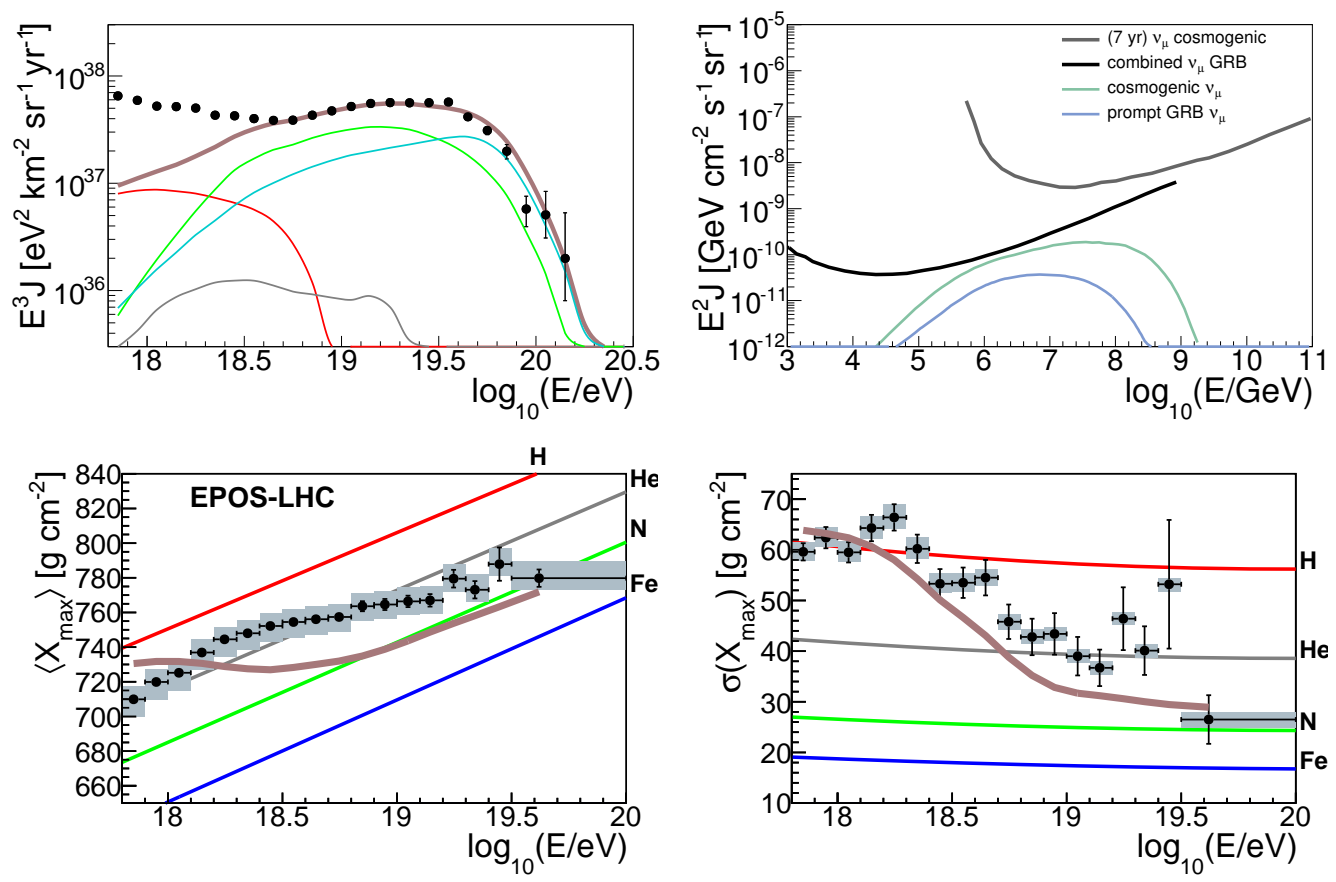

Figure 4: Figure taken from [3] - Cosmic-ray and neutrino observables obtained with the best-fit parameters in the Mixed Composition Ankle Model. Upper left: simulated energy spectrum of UHECR (multiplied by $E^{3}$ ) compared to data from [19]. Spectra at Earth are grouped according to the mass number as follows: $A=1$ (red), $2 \leq A \leq 4$ (grey), $5 \leq A \leq 22$ (green), $23 \leq A \leq 28$ (cyan), total (brown). Upper right: Prompt and cosmogenic (muon flavor) neutrino spectra. The differential limits are defined as in [6]. Bottom: average and standard deviation of the $X_{\max }$ distribution as predicted (assuming EPOS-LHC [23] for UHECR-air interactions) for the model versus pure $\left({ }^{1} \mathrm{H}\right.$ (red), ${ }^{4} \mathrm{He}$ (grey), ${ }^{14} \mathrm{~N}$ (green) and ${ }^{56} \mathrm{Fe}$ (blue)) compositions, compared to data from [24].

Observatory.

We discussed different regimes for the interactions of nuclei in a GRB shell, relative to the development of the nuclear cascade in the source and to the ejected cosmic rays and neutrinos, as a function of the shell parameters. We found that ejected UHECR spectra exhibit a rigidity-dependent maximal energy cutoff, which is an assumption that is often used in UHECR source models, only in the low-luminosity GRB case, where the photo-disintegration is not very efficient. Another remarkable result is that photo-meson production off nuclei constitutes the main contribution to the neutrino production in the Empty Cascade Case, and it is relatively less known with respect to the photomeson production off nucleons (happening mostly in the Populated Cascade and Optically Thick Case), requiring further study.

The ejected cosmic rays are propagated to the Earth and a fit of the Auger spectrum and composition is performed. If the UHECR transition to the GRB contribution occurs at the ankle, GRBs can describe cosmic ray and neutrino data, even with a pure injection composition (at the expense of relatively high baryonic loadings and with either low $\gamma$-ray luminosities or large collision radii as GRB parameters).

We have then demonstrated that a detailed computation of the interactions in the source and in the 
propagation, leading to neutrino fluxes, has the power to rule out a region of parameters related to characteristics of the source. Further developments of the fit method will improve the constraining power of the neutrino fluxes and similar procedures will be applied in the future to multi-zone models in GRBs and to different classes of sources.

Acknowledgments. We would like to thank our colleagues from the Pierre Auger Collaboration for fruitful discussions. This project has received funding from the European Research Council (ERC) under the European Union's Horizon 2020 research and innovation programme (Grant No. 646623).

\section{References}

[1] T. Piran, Rev. Mod. Phys. 76, 1143 (2004) [astro-ph/0405503].

[2] E. Waxman and J. N. Bahcall, Phys. Rev. Lett. 78, 2292 (1997) [astro-ph/9701231].

[3] D. Biehl et al., [arXiv:1705.08909], submitted to A\&A.

[4] A. Aab et al. [Pierre Auger Collaboration], Phys. Rev. D 90, no. 12, 122005 (2014), [arXiv:1409.4809].

[5] S. Hummer et al., Phys. Rev. Lett. 108, 231101 (2012) [arXiv:1112.1076]

[6] P. Baerwald et al., Astropart. Phys. 62, 66 (2015) [arXiv:1401.1820].

[7] P. Baerwald et al., Astrophys. J. 768, 186 (2013) [arXiv:1301.6163].

[8] P. Baerwald et al., Astropart. Phys. 35, 508 (2012) [arXiv:1107.5583].

[9] D. Boncioli et al., Scientific Reports 7, 4882 (2017) [arXiv:1607.07989].

[10] J. L. Puget et al., Astrophys. J. 205, 638 (1976).

[11] F. W. Stecker and M. H. Salamon, Astrophys. J. 512, 521 (1999) [astro-ph/9808110].

[12] A. J. Koning et al., Proceedings, International Conference on Nuclear Data for Science and Tecnology (2007), pp. 211-214.

[13] R. Alves Batista et al., JCAP 1510, no. 10, 063 (2015) [arXiv:1508.01824]

[14] A. Fedynitch et al., $\operatorname{PoS}(2017) 559$, this Conference

[15] R. Aloisio et al., JCAP 1210, 007 (2012) [arXiv:1204.2970].

[16] R. C. Gilmore et al., Mon. Not. Roy. Astron. Soc. 422, 3189 (2012) [arXiv:1104.0671].

[17] A. Aab et al. [Pierre Auger Collaboration], JCAP 1704, no. 04, 038 (2017) [arXiv:1612.07155].

[18] S. Shibata and N. Tominaga, Publ. Astron. Soc. Jap. 67, no. 3, 49 (2015) [arXiv:1503.03662]

[19] I. Valiño for the Pierre Auger Collaboration, PoS(2015)271, [arXiv:1509.03732].

[20] M. G. Aartsen et al. [IceCube Collaboration], Astrophys. J. 809, no. 1, 98 (2015) [arXiv:1507.03991].

[21] M. G. Aartsen et al. [IceCube Collaboration], [arXiv:1702.06868].

[22] M. G. Aartsen et al. [IceCube Collaboration], Phys. Rev. Lett. 117, no. 24, 241101 (2016) [arXiv:1607.05886].

[23] T. Pierog et al., Phys. Rev. C 92, no. 3, 034906 (2015) [arXiv:1306.0121].

[24] A. Porcelli for the Pierre Auger Collaboration, PoS(2015)420, [arXiv:1509.03732]. 